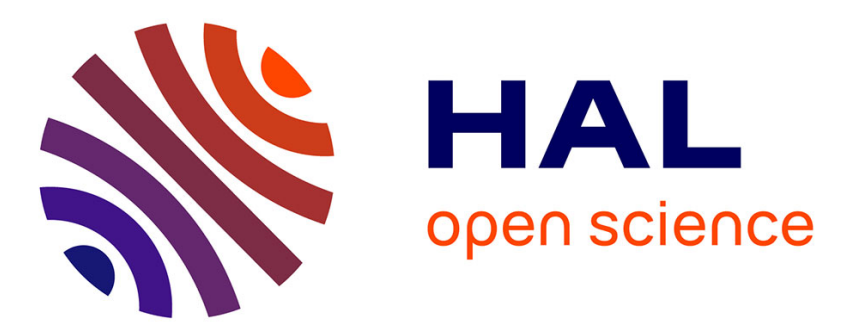

\title{
Bottom-up scientific field detection for dynamical and hierarchical science mapping - methodology and case study
}

David Chavalarias, Jean-Philippe Cointet

\section{- To cite this version:}

David Chavalarias, Jean-Philippe Cointet. Bottom-up scientific field detection for dynamical and hierarchical science mapping - methodology and case study. Scientometrics, 2008, 75 (1), pp.20. 10.1007/s11192-007-1825-6 . hal-00120697v3

\section{HAL Id: hal-00120697 \\ https://hal.science/hal-00120697v3}

Submitted on 23 Jan 2007

HAL is a multi-disciplinary open access archive for the deposit and dissemination of scientific research documents, whether they are published or not. The documents may come from teaching and research institutions in France or abroad, or from public or private research centers.
L'archive ouverte pluridisciplinaire HAL, est destinée au dépôt et à la diffusion de documents scientifiques de niveau recherche, publiés ou non, émanant des établissements d'enseignement et de recherche français ou étrangers, des laboratoires publics ou privés. 


\title{
Bottom-up scientific field detection for dynamical and hierarchical science mapping, methodology and case study
}

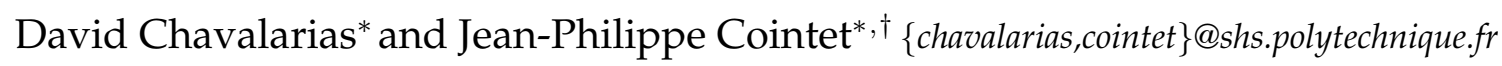

January 19, 2007

\begin{abstract}
We propose new methods in order to detect paradigmatic fields thanks to simple statistics over a scientific content database. We define an asymmetric paradigmatic proximity between concepts which provide hierarchical structure and test our methods on a case study with a database made of several millions of resources. We also propose overlapping categorisation to describe paradigmatic fields as sets of concepts that may have several different usages. Concepts can also be dynamically clustered provinding a high-level description of the evolution of the paradigmatic fields.
\end{abstract}

Keywords: Mapping and visualisation of knowedge ; publication analysis ; coword analysis ; paradigmatic evolution ; paradigmatic proximity.

\section{Introduction}

Modern acceptation of paradigm has been provided by KUHN (1970) as "an entire constellation of beliefs, values and techniques, and so on, shared by the members of a given community". He contended that, a paradigm enables a group of scientists to focus its efforts on a well-defined range of problems. A paradigm enables the scientific community to converge toward a consensus concerning the definition of important problems and identification of techniques needed to solve

\footnotetext{
${ }^{*}$ CREA (Center for Research in Applied Epistemology), CNRS-Ecole Polytechnique, 1 rue Descartes, 75005 Paris, France

${ }^{\dagger}$ TSV (Social and Political Transformations related to Life Sciences and Life Forms), INRA, 65 Boulevard de Brandebourg, 94205 Ivry-sur-Seine Cedex France
} 


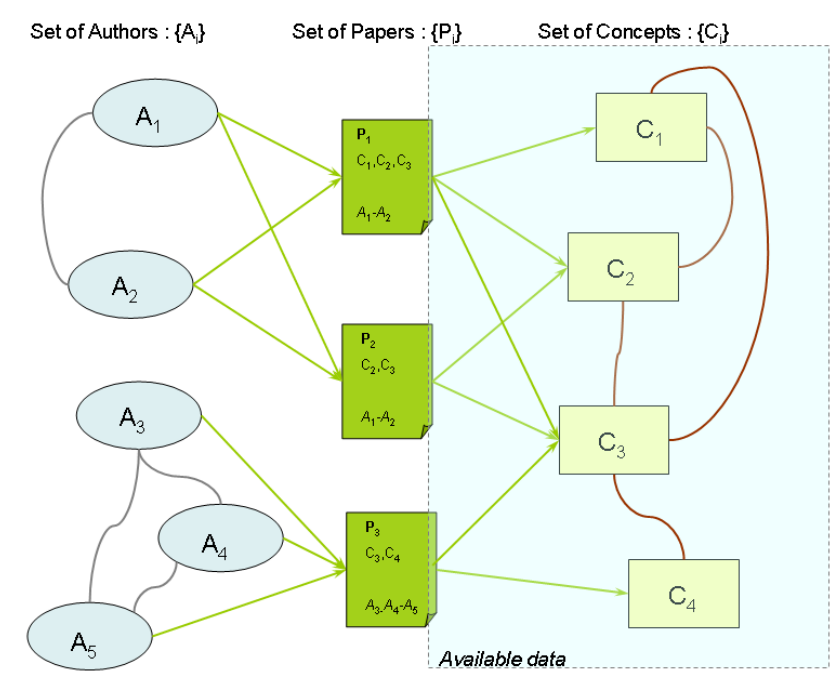

Figure 1: scientific knowledge production scheme : a set of authors $\left\{A_{i}\right\}$ produce publications $\left\{P_{i}\right\}$ which are made of concepts $\left\{C_{i}\right\}$. We defined paradigmatic field as strongly cooccurring set of concepts.

them, and last but not least for our purpose, which set of concepts shall be used to share their breakthrough. In the following we will call such sets paradigmatic fields.

The figure 1 represents a schematic view of scientific knowledge production. Authors $\left\{A_{i}\right\}$ publish papers $\left\{P_{i}\right\}$ that contain informative sets of concepts $\left\{C_{i}\right\}$. Some of these publications have been co-authored while some concepts may be strongly co-occurring with others. On this scheme, we linked authors that have co-authored an article, and concepts that have co-occurred in one paper at least. Our assumption is that paradigmatic fields found in public sphere of knowledge production provide a direct insight into the very structure of science and researchers communities. We claim that the conceptual side of this complex affiliation network is enough to describe the scientific field as an overlapping set of paradigmatic fields.

We shall then define paradigmatic field as a strongly cooccurring set of concepts which corresponds in graph theory as a dense subset of the conceptual network. Our example features two overlapping paradigmatic field, the first one is made of the set of concepts $\left\{C_{1}, C_{2}, C_{3}\right\}$, the second one is made of $\left\{C_{3}, C_{4}\right\}$. We will voluntarily disregard the collaboration side (on the left) in the following to concentrate on the conceptual network we built.

The aim of this paper is to present tools for automatic bottom-up identification of paradigmatic fields directly from articles database. The strength of our approach is that is does not require other information than the one already available in most existing database to dynamically 
reconstruct the multi-scale structure of paradigmatic fields. Rough statistics about occurrences and co-occurrences of words in indexed documents are sufficient. In particular, it does not require a real access to the content of each articles nor a particular linguistic treatment on words.

A simple measure of paradigmatic proximity henceforth noted $P_{p}$ is defined between a set of given key-words and is used to perform paradigmatic field detection. This bottom-up approach also aims at describing paradigmatic fields evolution through mere statistics on key-words occurrences and co-occurrences, over a 25 years period. First explanatory results are given.

Although the context here is the one of scientific knowledge production, the same method may be applied to get global insights of any kind of electronic database (patents, blogs or webpages, etc...)

\section{Context and rationale}

Scientometric research deals with study of science or technology using quantitative data. One of its prominent objective is the development of information systems that may help science studies practitioners or searchers to navigate into the outstanding mass of scientific papers published worldwide every day. A great number of methods for automatically designing conceptual maps have been proposed. DOYLE (1961) was one of the first to point to the fact that traditional document retrieval techniques are ineffective in finding relevant documents due to a lack of semantic understanding of relevance. Since then, several methods have been proposed to do intelligent scientific database management. The two main methods developed have been "citation-based analysis" and "co-word analysis". These methods are generally bottom-up which means that they do not need any supplementary information than lexical statistics of the articles database being surveyed.

Citation-based analysis can be of two kinds. On one hand "Bibliometric coupling" builds a similarity measure between documents according to the frequency with which two documents are cited together(SMALL, 1973; LEYDESDORFF \& VAUGHAN, 2006), on the other hand "bibliographical coupling" link preferentially document which share the same set of references (SALTON, 1963).

Co-word analysis usually tries to map concepts landscape using exclusively statistics about the number of co-occurrences of a word with another. A classical statistics in co-word analysis which has been extensively documented in literature (M. CALLON, 1983; CALLON ET AL., 1991; NOYONS \& VAN RAAN, 2002) is the similarity index measured as the ratio between the number of co-occurrences between word $a$ and $b$ divided by the product of the number of total occurrences 
of $a$ and $b$. Once this data has been collected clustering algorithms like kohonen maps algorithms are used to provide smarter navigation tools in articles databases thanks to conceptual mapping of a wide research area (LIN \& SOERGEL, 1991; SUN, 2004). Many approaches also propose to use both words occurrences and references to help producing knowledge maps(PETER VAN DEN BESSELAAR, 2006).

In our paper we claim that co-word analysis is a fruitful way to analyze massive scientific database. We show that it is possible to exhibit hierarchical structure in the basic original information with the sole help of statistics extracted from our original database. We explain our intuitive idea of paradigmatic proximity in the next section and explicit its formal expression in section 4. Our method is then tested on a very large scientific database (see section 5) before some preliminary results are given in the static and dynamical cases (section 6). We finally describe few perspectives related to our methodology.

\section{What can indexed scientific databases tell us about paradig- matic fields?}

It is now part of everyday life. When you want to find an article related to a concept $A$ you enter a request in your favorite search engine and get within a second the total number of papers dealing with this concept. To be more selective, you can refine your request to " $A$ AND $B$ ". At this point we can associate each concept with the set of articles that mention this concept. At this step, we have at least the two elementary tools of set theory : the number of articles that mention concept $A$ (set size) and the number of articles that contain both concept $A$ and concept $B(A \cap B)$. As we shall see, these two simple notions enable to define measures of paradigmatic proximity that are highly relevant to characterize paradigmatic fields. Moreover, since articles can be clustered by year of publication, it is possible to get the dynamics of the paradigmatic proximity that happens to be relevant to track the evolution of paradigms.

Let's illustrate our point with an example. On the figure 2 we plotted together occurrences and co-occurrences of "Public Goods", "Game theory" and "Experimental economics". "Game theory" and "Experimental economics" are both relevant concepts for the study of public goods. But the concept "experimental economics" is more specific than "game theory". Specific terms in game theory related to public goods would have been "ultimatum game", "prisoner's dilemma", etc... But this notion is not clear if we look only at co-occurrences from the point of view of "public goods": $P($ experimental economics $\mid$ Public goods) and $P($ game theory $\mid$ Public goods $)$ are of the 


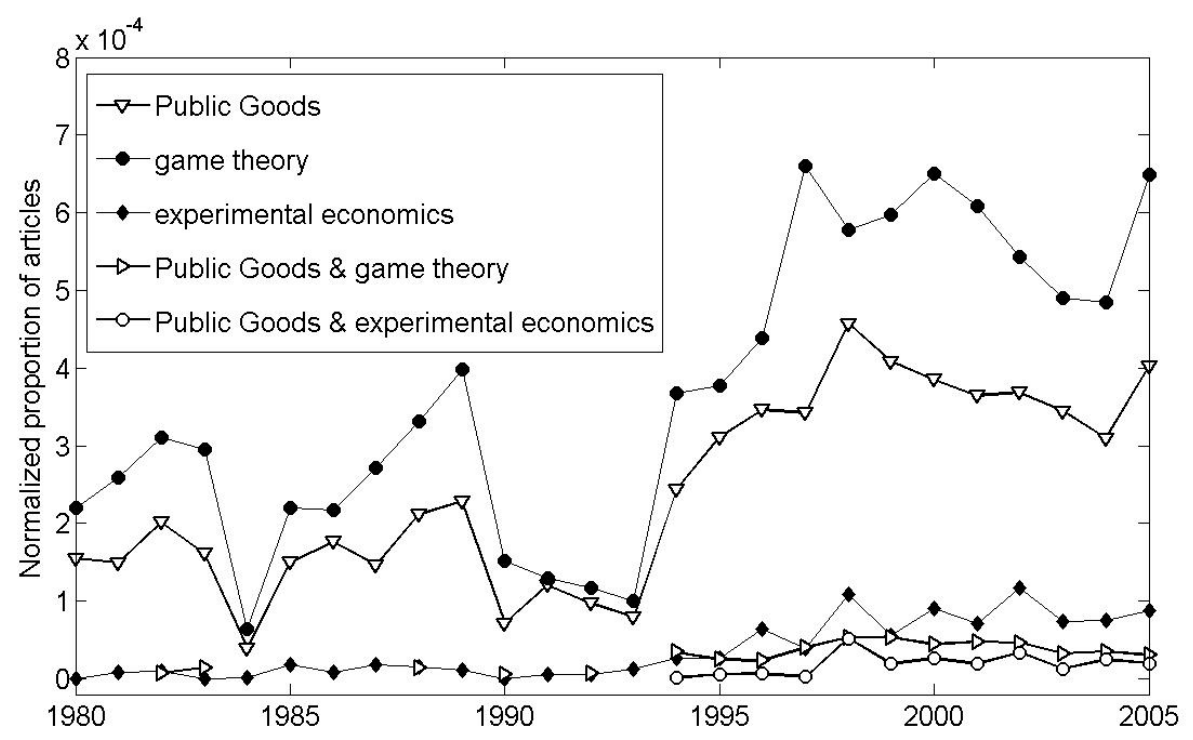

Figure 2: Comparative dynamics of occurrences and co-occurrences of concepts related to Public Goods

same order of magnitude. Then, if we switch the reference concept, $P($ Public goods|experimental economics) is much higher than $P$ (Public goods|game theory). This means that the concept of public goods is widely used in experimental economics studies but is less central in game theory. If we want to define a paradigmatic proximity that could distinguish "game theory" from "experimental economics" we should thus use the both kind of conditional probabilities. This notion of degree of specificity is important and suggests that we might want to have a parameter to tune the desired specificity.

Moreover, whereas a majority of papers in experimental economics deals with public goods, the reverse is not true and there are probably scientists working on public goods that never worked on experimental economics studies. The paradigmatic proximity should thus be asymmetric to reflect these kinds of situation.

We can summarize the different possible scenario that might be encountered as follows:

1. $P(A \mid B)$ high, $P(B \mid A)$ high : $A$ and $B$ are in the same paradigm and have about the same degree of specificity,

2. $P(A \mid B)$ low, $P(B \mid A)$ high : $B$ is general relatively to concept $A$ (e.g. A = public good and $\mathrm{B}=$ game theory),

3. $P(A \mid B)$ high, $P(B \mid A)$ low : $B$ belongs to a sub-domain relatively to $A$ (e.g. $\mathrm{A}=$ Game theory and B = public good),

4. $P(A \mid B)$ low, $P(B \mid A)$ low : $A$ and $B$ are weakly relevant to each other, 
We will now try to define a paradigmatic proximity such that it could be possible to discriminate the three first cases and eliminate the last one.

\section{Paradigmatic proximity definition}

Classical scientometric statistics uses number of concepts occurrences and co-occurrence in a given time window. Starting from an article database with $N$ articles, for given concepts $i$ and

$j$, let's note $n_{i}^{t}$ and $n_{j}^{t}$ the number of occurrences of $i$ and $j$ for the time window $t$ and $n_{i j}^{t}$ the number of co-occurrences for the same time range.

From the above, there are some properties that we wish our paradigmatic proximity $P_{p}$ to compel:

1. $P_{p}(i, j)=0$ if $n_{i j}^{t}=0$

2. $\lim _{\frac{n_{i j}^{t}}{n_{i}^{t} \rightarrow 0}}\left(P_{p}(i, j)\right)=0$

3. $P_{p}(i, i)=1$

4. $P_{p}(i, j)$ is growing with $n_{i j}^{t}$ as larger co-occurrences sets illustrate higher paradigmatic proximity. $P_{p}(i, j)=f\left(n_{i j}^{t}\right), f$ being a growing function.

5. $P_{p}(i, j)$ should depend on $n_{i}^{t}$ and $n_{j}^{t}$, so that if one of them is growing $P_{p}(i, j)$ will decrease. It follows that $P_{p}(i, j)=f\left(n_{i j}^{t}, n_{i}^{t}, n_{j}^{t}\right), f$ being a growing function according to its first coordinate and a decreasing function according to the two others.

6. Last, we will have to estimate the paradigmatic proximity on a representative sample of the set of articles in the fields (typically a collection of journals). Under the assumption that the sample is representative we want the estimation to be independent of the sample's size. This means that we also wish that semantic proximity between two words to be independent of the total number of articles in the database to be an homogeneous function of $n_{i j}^{t}, n_{i}^{t}, n_{j}^{t}$ i.e. $f(\lambda x, \lambda y, \lambda z)=f(x, y, z)$. From this property we deduce that we can write $f$ as a function of $n_{i j}^{t} / n_{i}^{t}$ and $n_{i j}^{t} / n_{j}^{t}$

When $n_{i j^{t}} \rightarrow 0$ we expect our distance to be null. Hence if we write the Taylor development of $P_{p}$ in 0 we should have : $P_{p}(x, y)=\alpha_{0}+\alpha_{11} x+\alpha_{12} y+\alpha_{21} x^{2}+\alpha_{22} y^{2}+\alpha_{23} x y+$ $\alpha_{31} x^{3}+\alpha_{32} y^{3}+\alpha_{33} x y^{2}+\alpha_{34} x^{2} y+\ldots$. From assumption 2) we can deduce that $\alpha_{0}=0, \alpha_{11}=$ $\alpha_{12}=0$ and so on.... Hence $P_{p}$ can be written as the sum of crossed products : $P_{p}\left(\frac{n_{i j}^{t}}{n_{i}^{t}}, \frac{n_{i j}^{t}}{n_{j}^{t}}\right)=$ $\Sigma_{i=1}^{\infty} \Sigma_{j=1}^{i-1} \alpha_{i j}\left(n_{i j}^{t} / n_{i}^{t}\right)^{j}\left(n_{i j}^{t} / n_{j}^{t}\right)^{i-j}$.

The simplest class of functions that fit this Taylor development in 0 as well as all the above conditions are the Cobb-Douglas functions $f_{\alpha, \beta}(x, y)=x^{\alpha} y^{\beta}$. Moreover we know from the 
previous condition that $\mathrm{f}$ is growing, consequently $a>0$ and $b>0$. We thus decide to define the paradigmatic proximity by :

$$
P_{p}^{\alpha, \beta}(i, j)=\left(n_{i j}^{t} / n_{i}^{t}\right)^{\alpha}\left(n_{i j}^{t} / n_{j}^{t}\right)^{\beta}
$$

From this expression, it is straightforward to see that given a concept $i$ an looking for the closest concepts $j$ :

- $1>>\alpha>0$ will favor concepts $j$ such that $P(j \mid i)$ is low,

- $\beta>>1$ will favor concepts $j$ such that $P(i \mid j)$ is low,

For $\alpha=1$ and $\beta=1$, the paradigmatic proximity has an intuitive interpretation: it is proportional to the probability that an article contain both concepts $i$ and $j$ in the database $\left(\frac{n_{i j}}{N}\right)$ over the probability that an article would contain both concepts $i$ and $j$ if co-occurrences of $i$ and $j$ where random $\left(\frac{n_{i}}{N} \cdot \frac{n_{j}}{N}\right)$. The classical similarity index is thus a particular case of our paradigmatic measure for $\alpha=\beta=1$.

In this article, we will focus on the relations of paradigmatic proximity qualified by "specificity" or "generalization", i.e. on cases 2 and 3. To limit the parameter space, we will reduce our investigations to a parameterized expression of $P_{p}^{\alpha, \beta}$ noted $P_{p}^{\alpha}$ with $\alpha>0$. Given the remarkable symmetrical proximity for $\alpha=\beta=1$ the condition we choose is that $P_{p}^{\alpha}(i, j)=P_{p} \frac{1}{\alpha}(j, i)$ i.e. if a concept $j$ is qualified as more specific from the point of view of $i$ (case 3), then changing $\alpha$ for $\frac{1}{\alpha}$ will enable to detect concept $i$ as a general neighbor from the point of view of $j$ (case 2) the values of paradigmatic proximities being the same in both cases.

We will thus further consider the sub-class of function :

$$
P_{p}^{\alpha}(i, j)=\left(n_{i j}^{t} / n_{i}^{t}\right)^{\alpha}\left(n_{i j}^{t} / n_{j}^{t}\right)^{1 / \alpha}
$$

As we shall see, this distance will enable to describe the way a concept belongs to a sub-field of a target concept or on the contrary how a target concept belongs to a sub-field of another concept.

We will now use this paradigmatic proximity measure to explore a given set of concepts with two different approaches. The first one can be defined as concept-centered. We will study neighborhoods of concepts in function of $\alpha$ (specific or generic paradigmatic proximity). At low value of $\alpha$, we catch the most precisely expressions near our target concept. When rising up $\alpha$, we access to more generic expressions. The second approach is a global mapping of the scientific field treated. We designed methods to describe dynamics of high level properties such 
as community structure.

\section{Methodology}

The case study presented here focuses on a set of concepts coming from two data sources : a set of keywords for complex systems field associated with European projet in IST Cordis's database from FP6 and FP7 (765 keywords generously provided by the Arc System team lead by Joseph Fröhlish - see appendix for the collection protocol) ; a set of keywords collected near colleagues (about one hundred). We got a partnership with Elsevier's search engine society (Scirus.com) in order to collect the number of occurrences and co-occurrences per year of theses concepts from 1975 to 2005 in the full text of the articles. The database gathered more than 20.000 .000 indexed articles.

To collect necessary statistics in a reasonable time we first had to restrain our set of concepts to 448 keywords (which are given in appendix). Since co-occurrences are very demanding in terms of server availability, we also decided to do a query on a co-occurrence only if the two queries on single terms gave a non zero result for authors keywords (each concept has been mentioned by at least one author as an article's keywords for the year considered). Consequently our database is built on all query results for single terms in full text from 1975 to 2005, and every query results on full text co-occurrences for couples of concepts that both appeared at least once as author keywords the year considered.

This database enables to compute the paradigmatic proximity for any time window from 1975 to 2005 . If we choose a time range between years $Y 1$ and $Y 2$, we thus have the following extended formulation of paradigmatic proximity:

$$
P_{p}^{\alpha}\left(i, j,\left[Y_{1} \ldots Y_{2}\right]\right)=\left(\frac{\sum_{t=Y_{1} \ldots Y_{2}}\left(n_{i j}^{t}\right)}{\sum_{t=Y_{1} \ldots Y_{2}} n_{i}^{t}}\right)^{\alpha}\left(\frac{\sum_{t=Y_{1} \ldots Y_{2}} n_{i j}^{t}}{\sum_{t=Y_{1} \ldots Y_{2}} n_{j}^{t}}\right)^{\frac{1}{\alpha}}
$$

We will now give some examples of application of our paradigmatic proximity measure. It should not be forgotten that the clusters and thematic fields that we will exhibit are conditional to our database of 448 concepts. There could be some more relevant concepts for the reader that will not be found because of the database incompleteness. 


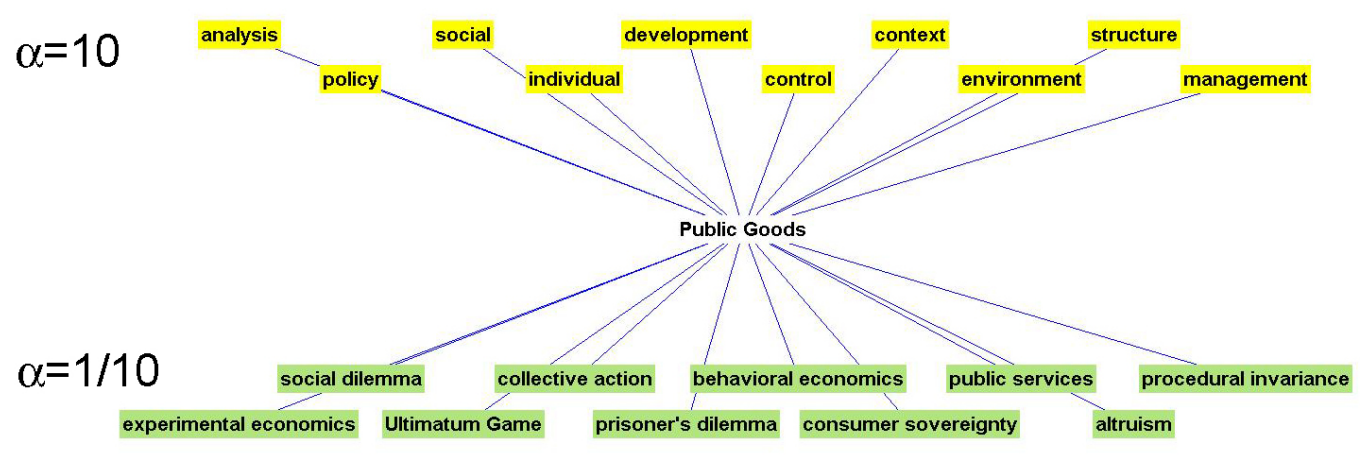

Figure 3: Two kinds of neighborhood of concept Public Goods. Inferior part: $\alpha=10$, the 10 closest concepts that specify Public Goods ; superior part: $\alpha=0.1$, the 10 closest concepts that are more generic than Public Goods.

\section{Case Study}

\subsection{Paradigmatic neighborhoods}

Our paradigmatic proximity enables to define neighborhood of a target concept $i$ given a threshold $s$ and an $\alpha$ value at time t by:

$$
V_{s, \alpha}^{t}(i)=\left\{j \mid P_{p}^{\alpha}(i, j, t)>s\right\}
$$

This neighborhood structure defined for each value of a outline relations of specification or generalization. On the example of public goods (cf. Figure 3 ), we can see that as $\alpha$ increases, words in a the neighborhood of public goods become more specific and closer to the concepts used by specialists of the fields. We thus get concepts that sharply qualify areas of investigations about public goods). Note that such a visualisation could also be used to navigate in a concept map with specific tools to zoom in or zoom out according to the specificity or generality of concepts searched.

\subsection{Identification of paradigmatic fields}

Once we have defined a similarity measure, and a neighborhood, we can try to draw knowledge map which is a common goal in scientometric literature (BUTER \& NOYONS, 2002; MARSHAKOVASHAIKEVICH, 2005). Looking at the bottom part of figure 3, we can see that it seems to coexist two distinct spheres of knowledge that use the concept "public goods". The first usage is rather "game theory" oriented, as the other is rather used as a political science concept. For example, Public Goods is linked to procedural invariance and to collective action but there no studies mention- 


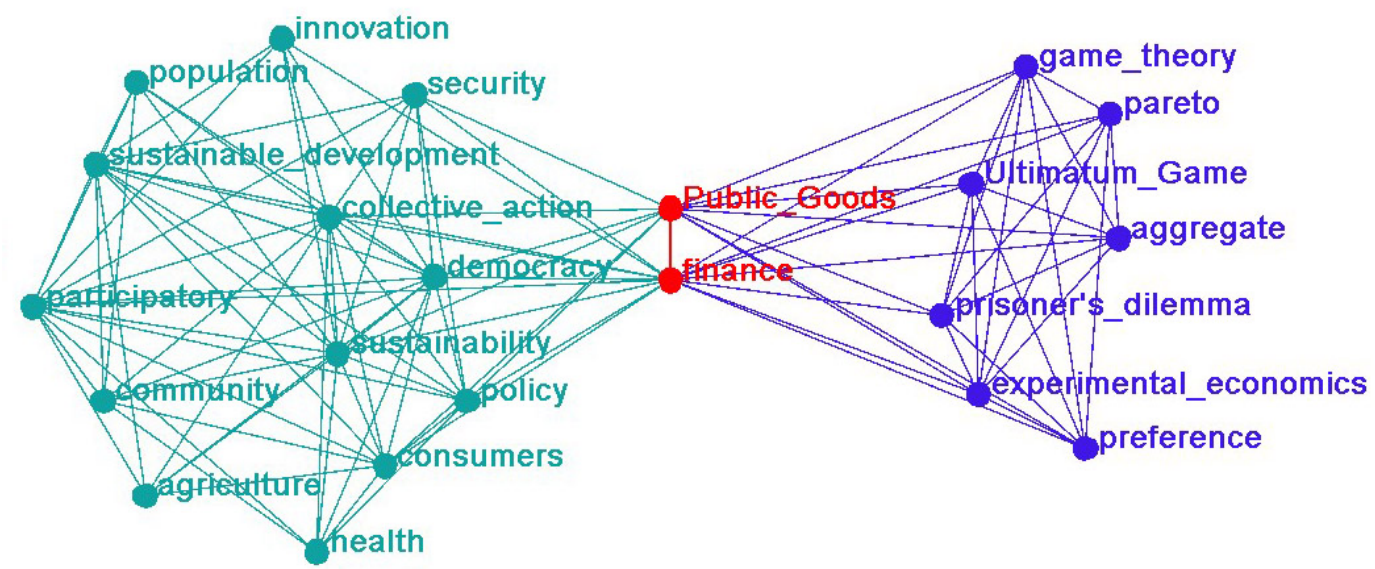

Figure 4: The two paradigmatic fields of public goods. Public Goods (as well as finance) belongs to two spheres of knowledge production, one game theory oriented, the other political sciences oriented.

ing both collective action and procedural invariance. These two concepts belong to two different spheres of knowledge production. Contextual information enables us to exhibit automatically these multiple usage.

To automatically exhibit these multiple usages and identify set of concepts reflecting scientific activity, we need a broader view of the conceptual landscape taking into account the relations between the different concept's neighbors. Given an $\alpha$ value, we need to categorize our data according to the values of the paradigmatic proximity $P_{p}^{\alpha}$. Since a word can have several meanings and can be used in several scientific communities, the categorization algorithm should make it possible for a word to belong to several different clusters. One successful method in line with this requirement is the clique percolation algorithm (PALLA ET AL., 2005) that operates on graphs of concepts to detect communities. Hence we generate a concepts graph based on our proximity measure by fixing a threshold $s$ and linking each concept $i$ to its set of neighbors: $V_{s, \alpha}(i)$. To avoid linking very generic words to any words we limit the maximum number of neighbors to 30 , taking the 30 closest when neighborhood size is superior. This enables to build a non-directed graph on concepts. Then we can apply the k-clique percolation algorithm which outlines communities of concepts that qualify distinct spheres of knowledge production. We illustrate this overlapping categorization displaying the to paradigmatic fields identified around public goods in the period 2003-2005 (cf. fig. 4).. We observe that it indeed belong to two communities in our concepts set.

It shoud be emphasized here that this visualisation is complementary to the one of neighborhoods. Here only neighbors that satisfies global conditions appear. Thus the detected fields 
outline trends in science, associated to a degree of specificity tuned by $\alpha$.

\subsection{Dynamics of paradigmatic fields}

$$
\begin{gathered}
\text { pareto } \\
\text { prisoner's dilemma } \\
\text { general equilibrium } \\
\text { public services } \\
\text { collective action } \\
\text { economic models } \\
\text { game theory } \\
\text { altruism } \\
\text { experimental economics } \\
\text { social dilemma } \\
\text { behavioral economics } \\
\text { bounded rationality } \\
\text { Ultimatum Game } \\
\text { selfish } \\
\text { democracy } \\
\text { framing effect } \\
\text { consumer sovereignty } \\
\text { heterogeneous agents } \\
\text { conformism } \\
\text { procedural rationality } \\
\text { consumer behavior } \\
\text { procedural invariance } \\
\text { collective rationality }
\end{gathered}
$$

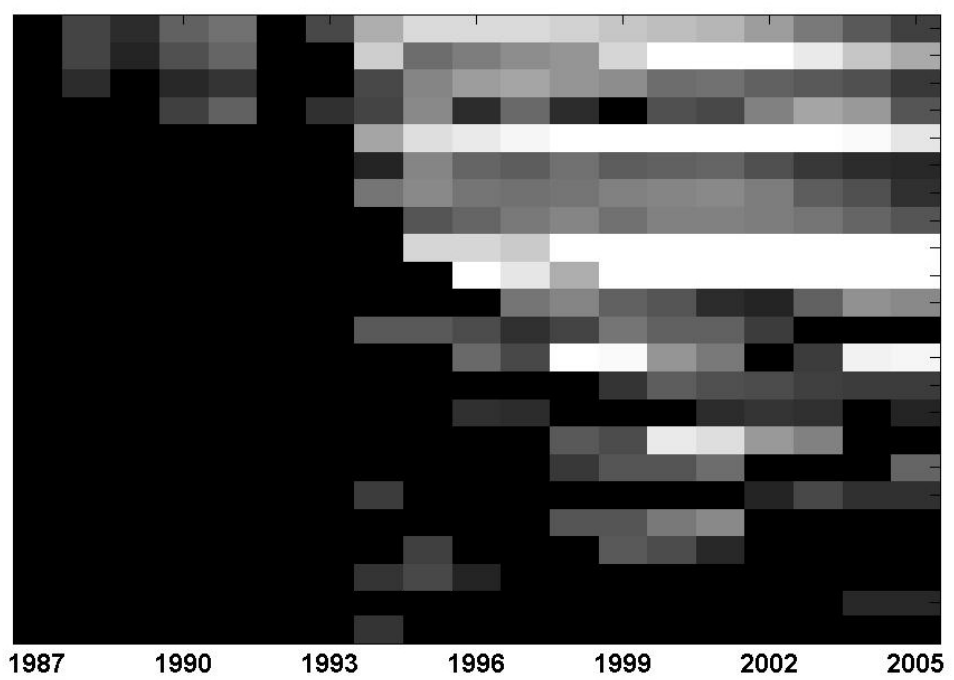

Figure 5: Dynamical view of the evolution of the paradigmatic fields around Public goods from 1987 to 2005 (each year corresponds to the aggregation of a 3-years time-window) for $\alpha=1$. A black box means that the concept was below the threshold at the considered year. The lighter the square, the higher the paradigmatic proximity. We can see that public goods studies appraised as game theory issue developed a lot these last years. Among emerging close concepts in the fields we find heterogenous agents and procedural rationality. These observations fit well with what we actually observe in evolution of public goods studies.

Dynamical science mapping is another challenge that aim at describing dynamical patterns in science evolution (GARFIELD, 2004; BRAAM ET AL., 1991). Static visualization based paradigmatic proximity is only partially informative. Our temporal time series enable us to study evolution of paradigmatic proximity and paradigmatic fields through time. Several questions arise : is it possible to reconstruct the historical evolution of major paradigmatic shifts, can we detect automatically emerging approaches and sub-fields? The simplest way to take into account the dynamical dimension of our data is to represent the evolution of paradigmatic neighborhoods through time. Given a target word and a threshold $s$, we can plot for each time-window $t$ the set of words belonging to the neighborhood $V_{s, \alpha}^{t}(i)$ as given figure 3 . We can thus provide dynamical evolution of a target concept's evolution as illustrated 5 . 


\section{Perspective}

We have already sketched methods to provide high-level description of our set of concepts. The next step may be to integrate time related data in this high-level description, in order to have a dynamical evolution of the paradigmatic fields.

Another challenge is to reintroduce the directionality in the high-level description we developed. The sets of concepts grouped with the clustering algorithm we used is reduced to a flat description. Yet our original data exhibited asymmetric relations between concepts according to the value of $\alpha$. It would be highly relevant to describe cohesive paradigmatic field not only as clouds of concepts but as a three dimensional structure by including the dimension illustrated fig 3.

\section{Conclusion}

Massive collections of scientific publications are now available on-line thanks to multiple public platforms. These databases usually cover large-scale scientific production over several decades and for a broad range of thematic areas. Today researchers are used to perform queries on these databases with keywords or combination of keywords in order to find articles associated to a precise scientific field. This full text indexation performed for millions of articles represents a huge amount of public information. But instead of being used to characterize articles, can we revert the standpoint and use this information to characterize concepts neighborhood and their evolution? In this paper we give a yes answer to this question looking more precisely at the way concepts can be dynamically clustered to shed light on the way paradigm are structured.

Acknowledgements This study was supported by the CREA - Ecole Polytechnique, the ISTFET coordinated action ONCE-CS and the Paris Ile-deFrance Institute for Complex Systems. We warmly thanks the Scirus company for its partnership and Scott Craig for his kind help with the data processing, as well as Arc System research for their keywords list.

\section{References}

BraAm, R. R., Moed, H. F., VAn RaAn, A. F. J. (1991), Mapping of science by combined cocitation and word analysis. II. dynamical aspects, Journal American Society Information Science, 
42(4):252-266.

Buter, R., NOyOnS, E. (2002), Using bibliometric maps to visualise term distribution in scientific papers, in: Sixth International Conference on Information Visualisation (IV'02), pp. 697-702.

Callon, M., Courtial, J., Laville, F. (1991), Co-word analysis as a tool for describing the network of interaction between basic and technological research: The case of polymer chemistry, Scientometric, 22(1):155-205.

DoYLE, L. B. (1961), Semantic road maps for literature searchers, J. ACM, 8(4):553-578.

GARFIELD, E. (2004), Historiographic mapping of knowledge domains literature, Journal of Information Science, 30(2):119-145.

KUHN, T. S. (1970), The Structure of Scientific Revolutions, UCP, Chicago, second edition.

LEYDESDORFF, L., VAUGHAN, L. (2006), Co-occurrence matrices and their applications in information science: Extending aca to the web environment, J. Am. Soc. Inf. Sci. Technol., 57(12):1616-1628.

LIN, X., SOERGEL, D. (1991), A self organizing semantic map for information retrieval, Proc. 14th International SIGIR Conference:262-269.

M. CALLON, J. C., S. BAUIN (1983), From translation to problematic networks: an introduction to coword analysis, Social Science Information, 22:191-235.

MARSHAKOVA-SHAIKEVICH, I. (2005), Bibliometric maps of field of science, Infometrics, 41(6):1534-1547.

Noyons, E., VAn RAAn, A. (2002), Dealing with the data flood. Mining data, text and multimedia., J. Meij (ed.), The Hague: STT/Beweton, pp. 64-72.

PAlla, G., Derenyi, I., FARKAS, I., VicSeK, T. (2005), Uncovering the overlapping community structure of complex networks in nature and society, Nature, 435:814.

Peter VAn Den BesselaAR, G. H. (2006), Mapping research topics using word-reference cooccurrences: A method and an exploratory case study, Scientometrics, 68(3):377-393.

SALton, G. (1963), Associative document retrieval techniques using bibliographic information, J. ACM, 10(4):440-457. 
SMALL, H. G. (1973), Co-citation in the scientific literature: A new measure of the relationship between two documents, Journal of American Society for Information Science, 24(4):265-269.

SUN, Y. (2004), Methods for automated concept mapping between medical databases, J. of Biomedical Informatics, 37(3):162-178. 